\title{
Effects of augmentation agents in autistic disorder patients treated with risperidone: a systematic review and a meta- analysis
}

\author{
Efeito de estratégias de potencialização da risperidona em pacientes com \\ transtorno do espectro autista: revisão sistemática e metanálise
}

Different studies highlight the importance of augmentative strategies to risperidone as an effective pharmacological approach for treating behavioral symptoms associated with autistic spectrum disorder (ASD). ${ }^{1}$ The objective of the present study was to assess the combined clinical effects of different addon pharmacological therapies to risperidone in the management of patients with ASD.

After searching MEDLINE and EMBASE databases, we performed a systematic review following the PRISMA statement guidelines. ${ }^{2}$ We also looked for controlled trials by contacting experts and searching the website clinicaltrials.gov. The criteria used to select articles were: 1) English language; 2) randomized, controlled trials; 3) mean and standard deviation values provided; and 4) response and remission rates provided. We excluded controlled trials assessing therapies different from our study objective. We opted to use Hedges' $g$ to measure the effect size as it is appropriate for small sample sizes. The pooled effect size was weighted by the inverse variance method and measured under the random effect model. Heterogeneity was assessed using $\mathrm{I}^{2}$ index. We further used funnel plots to check for the existence of publication bias.

A total of 6 out of 51 randomized controlled trials met the eligibility criteria $(n=231$, mean age $=8.25$ years) ${ }^{3-9}$ (Table 1 ). In each trial reporting autistic symptoms, patients were clinically assessed by the Aberrant Behavior Checklist - Community version $(A B C-C) .{ }^{10}$ Association of drugs with risperidone included: amantadine, $\mathrm{N}$-acetylcysteine, topiramate, pentoxifylline, riluzole, and celecoxib.

Considering continuous reductions of autistic symptoms based on $\mathrm{ABC}-\mathrm{C}$ scores, we found a significant clinical difference between the active control group and the placebo group for the irritability domain (Hedge's $\mathrm{g}=1.23 ; 95 \%$ confidence interval [95\%CI] $0.825-$ $1.641 ; \mathrm{p}=0.000$ ) and lethargy domain (Hedge's $\mathrm{g}=$ $0.735 ; 95 \%$ CI $-0.163-1.307 ; p=0.02$ ). No significant difference was found for hyperactivity (Hedge's $\mathrm{g}=$ $0.651 ; 95 \%$ CI $-0.098-1.402 ; p=0.08$ ) or stereotypic movements (Hedge's g $=0.892 ; 95 \% \mathrm{CI}-0.021-1.806$; $p=0.056)$ (Figure 1 ).

Table 1 - Demographic characteristics and clinical protocols

\begin{tabular}{|c|c|c|c|c|c|c|c|}
\hline Author & Year & $\begin{array}{c}\text { Placebo } \\
\text { (n) }\end{array}$ & $\begin{array}{l}\text { Active } \\
\text { (n) }\end{array}$ & $\begin{array}{c}\text { Mean age } \\
(y)\end{array}$ & $\begin{array}{c}\text { Augmentation } \\
\text { strategy }\end{array}$ & $\begin{array}{c}\text { Active dosage } \\
(\mathrm{mg} / \mathrm{d})\end{array}$ & $\begin{array}{c}\text { Risperidone } \\
\text { dosage }(\mathrm{mg} / \mathrm{d})\end{array}$ \\
\hline Mohammadi et al. ${ }^{10}$ & 2013 & 20 & 20 & 8 & Amantadine & $100-150$ & $1-2$ \\
\hline Ghanizadeh et al. ${ }^{8}$ & 2013 & 17 & 14 & 9.5 & NAC & 0.76 & 0.92 \\
\hline Rezaei et al. ${ }^{4}$ & 2010 & 20 & 20 & 7.5 & Topiramate & 150 & 2.5 \\
\hline Akhondzadeh et al. ${ }^{3}$ & 2010 & 20 & 20 & 8 & Pentoxifylline & 500 & 2.5 \\
\hline Ghaleiha et al. ${ }^{7}$ & 2013 & 20 & 20 & 8.5 & Riluzole & 1.2 & 1.6 \\
\hline Asadabadi et al. ${ }^{5}$ & 2013 & 20 & 20 & 8 & Celecoxib & 300 & 3 \\
\hline
\end{tabular}




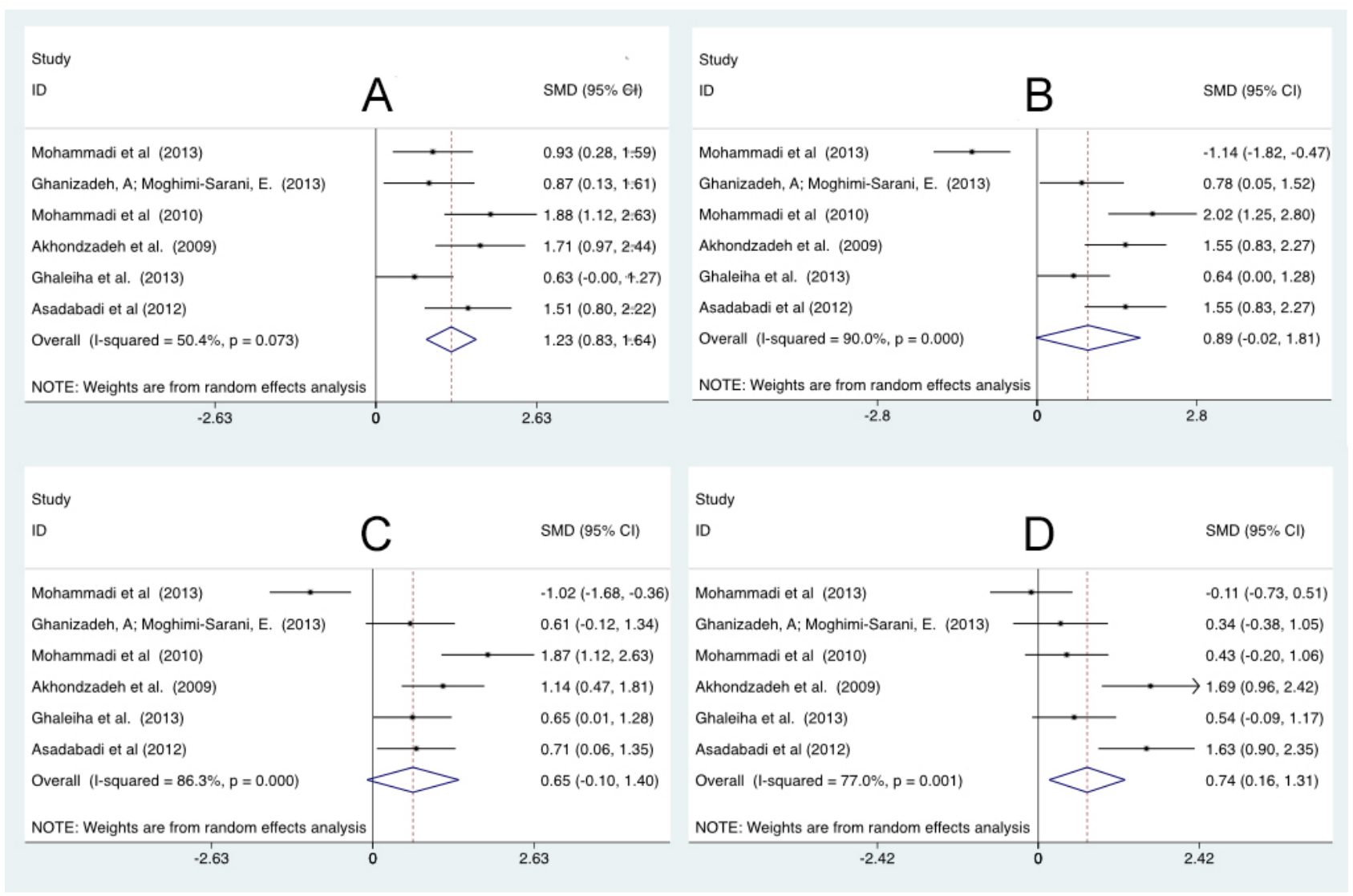

Figure 1 - Forest plot of effect sizes (Hedges' g) for active versus placebo group treatment: A) irritability; B) hyperactivity; C) stereotypic behavior; D) lethargy. $95 \% \mathrm{CI}=95 \%$ confidence interval; SMD = standardized mean difference.

Statistical analysis underscored the heterogeneity among studies ( $\mathrm{I}^{2}:$ 50.4\%). Meta-regression analysis for possible confounding factors - such as baseline severity scores, age, sample size, and augmentation strategy - revealed no significant correlation with effect size. All studies were within the limits of Begg \& Mazumdar's rank correlation test. Moreover, no study individually influenced the pooled effect size as assessed by the "metainf" command in Stata. In other words, our results suggested no difference between augmentation strategies in the subgroup analysis.

We found augmentation strategies to risperidone to be effective for amelioration of both irritability and lethargy in autistic patients. However, some limitations of the study - such as small number of trials and high heterogeneity - are factors that compromise its external validity. Nevertheless, we believe that the results presented underscore the benefits of combined therapy in comparison to risperidone alone and may work as a hypothesis-driven scenario for further clinical trials. The effectiveness of augmentation treatments to risperidone and the appropriate robustness of the strategy for treating autism are still unclear.
Augmentation strategies are promising tools for ameliorating both irritability and lethargy in patients with ASD. Further trials with larger samples will help to clarify the precise effects of augmentation strategies for this population.

\section{Amanda Soares, ${ }^{1}$ Pedro Shiozawa, ${ }^{1}$ Alisson Paulino Trevizol, ${ }^{1}$ Cristiane Silvestre de Paula, ${ }^{2}$ Rosane Lowenthal, ${ }^{1}$ Quirino Cordeiro ${ }^{1}$}

${ }^{1}$ Laboratório de Neuromodulação Clínica, Faculdade de Ciências Médicas da Santa Casa de São Paulo, SP, Brazil. ${ }^{2}$ Universidade Mackenzie, São Paulo, SP, Brazil.

\section{References}

1. Barnard L, Young AH, Pearson J, Geddes J, O'Brien G. A systematic review of the use of atypical antipsychotics in autism. J Psychopharmacol. 2002;16:93-101.

2. Liberati A, Altman DG, Tetzlaff J, Mulrow C, Gotzsche PC, Ioannidis 'JP, et al. The PRISMA statement for reporting systematic reviews and meta-analyses of studies that evaluate health care interventions: explanation and elaboration. PLoS Med. 2009;6:e1000100.

3. Akhondzadeh S, Fallah J, Mohammadi MR, Imani R, Mohammadi $M$, Salehi $B$, et al. Double-blind placebo-controlled trial of pentoxifylline added to risperidone: effects on aberrant behavior in children with autism. Prog Neuropsychopharmacol Biol 
Psychiatry. 2010;34:32-6

4. Rezaei V, Mohammadi MR, Ghanizadeh A, Sahraian A, Tabrizi M, Rezazadeh SA, et al. Double-blind, placebo-controlled trial of risperidone plus topiramate in children with autistic disorder. Prog Neuropsychopharmacol Biol Psychiatry. 2010;34:1269-72.

5. Asadabadi M, Mohammadi MR, Ghanizadeh A, Modabbernia A Ashrafi M, Hassanzadeh E, et al. Celecoxib as adjunctive treatment to risperidone in children with autistic disorder: a randomized, double-blind, placebo-controlled trial. Psychopharmacology (Berl). 2013;225:51-9.

6. Ghaleiha A, Ghyasvand M, Mohammadi MR, Farokhnia M, Yadegari $\mathrm{N}$, Tabrizi $\mathrm{M}$, et al. Galantamine efficacy and tolerability as an augmentative therapy in autistic children: a randomized, doubleblind, placebo-controlled trial. J Psychopharmacol. 2013;28:67785 .

7. Ghaleiha $A$, Mohammadi $E$, Mohammadi MR, Farokhnia M, Modabbernia A, Yekehtaz $\mathrm{H}$, et al. Riluzole as an adjunctive therapy to risperidone for the treatment of irritability in children with autistic disorder: a double-blind, placebo-controlled, randomized trial. Paediatr Drugs. 2013;15:505-14.

8. Ghanizadeh A, Moghimi-Sarani E. A randomized double blind placebo controlled clinical trial of $\mathrm{N}$-Acetylcysteine added to risperidone for treating autistic disorders. BMC Psychiatry. 2013;13:196.

9. Mohammadi MR, Yadegari N, Hassanzadeh E, Farokhnia M, Yekehtaz H, Mirshafiee O, et al. Double-blind, placebo-controlled trial of risperidone plus amantadine in children with autism: a 10week randomized study. Clin Neuropharmacol. 2013;36:179-84.
10. Aman MG, Singh NN, Stewart AW, Field CJ. The aberrant behavior checklist: a behavior rating scale for the assessment of treatment effects. Am J Ment Defic. 1985;89:485-91.

\section{Correspondence:}

Pedro Shiozawa

Departamento de Psiquiatria, Faculdade de Ciências Médicas da Santa Casa de São Paulo

Rua Major Maragliano, 241, Vila Mariana

04017-030 - São Paulo, SP - Brazil

Tel.: +55 (11) 3466.2105

E-mail: pedroshiozawa@gmail.com

Submitted Oct 12 2015, accepted for publication Dec 152015. No conflicts of interest declared concerning the publication of this article.

Suggested citation: Soares A, Shiozawa P, Trevizol AP, de Paula CS, Lowenthal R, Cordeiro Q. Effects of augmentation agents in autistic disorder patients treated with risperidone: a systematic review and a meta-analysis [letter]. Trends Psychiatry Psychother. $2016 ; 38(2): 114-116$. http://dx.doi.org/10.1590/22376089-2015-0068 